\title{
PRÉSENTATION DE TROIS TEXTES DU LIBERTAIRE ÂNGELO JORGE PUBLIÉS DANS LA REVUE AMANHÃ
}

\author{
Apresentação de três textos do libertário Ângelo \\ Jorge, publicados na revista Amanhã
}

João Carlos Pereira*

\begin{abstract}
RESUMO
O objetivo deste trabalho é apresentar três textos visivelmente ignorados pelos especialistas de Ângelo Jorge, autor do romance utópico Irmânia (1912), e completar a sua biobibliografia publicada num artigo de 2006 da revista eletrónica E-Topia. Trata-se de um soneto de temática operária e de dois artigos de carácter político publicados em 1909 na efémera revista libertária Amanhã. A sua publicação nesse ano corresponde bem à fase anarquista de Ângelo Jorge que cresceu no Brasil. Estes três textos têm o interesse de abordar os principais assuntos que mobilizavam os anarquistas e de evidenciar a relação existente entre a obra literária e o ideário de Ângelo Jorge.

Palavras-chave: Ângelo Jorge; literatura e ideologia; revista libertária Amanhã
\end{abstract}

\section{RÉSUMÉ}

Le but de ce travail est de présenter trois textes visiblement ignorés des spécialistes d'Ângelo Jorge, auteur du roman utopique Irmânia (1912), et de compléter sa biobliographie parue dans un article de 2006 de la revue électronique E-Topia. Il s'agit d'un sonnet à thématique ouvrière et de deux articles à caractère politique publiés en 1909 dans l'éphémère revue libertaire Amanhã. Leur publication cette année-là correspond

* $\quad$ Universidade Lumière - Lyon 2. 
bien à la phase anarchiste d'Ângelo Jorge qui a grandi au Brésil. Ces trois textes offrent l'intérêt d'aborder les principaux sujets qui mobilisaient les anarchistes et de mettre en évidence le lien existant entre l'œuvre littéraire et les idées d'Ângelo Jorge.

Mots clés: Ângelo Jorge; littérature et idéologie; revue libertaire Amanhã.

\begin{abstract}
The goal of this work is to present three texts visibly ignored by experts of Ângelo Jorge, author of the utopic novel Irmânia (1912), and to complete his bibliography, published in a 2006 article in the on-line journal, E-Topia. The three works include a proletariat-themed sonnet and two political articles published in 1909 in the short-lived libertarian journal Amanhã. Their publication that year coincides with the anarchistic phase of Ângelo Jorge, who grew up in Brasil. These three texts allow us to explore the principal themes which mobilized the anarchists and brings to light the connection between a work of literature and the ideas of Ângelo Jorge.
\end{abstract}

Keywords: Ângelo Jorge; literature and ideology; libertarian magazine Amanhã.

\title{
1. La ReVUe anARchiste $A$ manHẽ (1909)
}

L'objectif de cet article est d'approfondir ce que nous avons écrit, dans une étude sur le roman Irmânia (cf. PEREIRA, 2013, p. 165-215), au sujet de trois textes d'Ângelo Jorge publiés en 1909 dans la revue Amanhã. Outre cette utopie littéraire dont la parution a eu lieu au moment où le mouvement anarchiste atteignait son apogée au Portugal, Ângelo Jorge a publié notamment des ouvrages à caractère didactique ou sociopolitique, comme Ginástica Mental das Crianças, en 1904, ou Olhando a Vida. Apontamentos de Crítica Social, en 1910, ou encore A Questão Social e a Nova Ciência de Curar, en 1912. Il est également l'auteur de recueils de poésie, d'un recueil de nouvelles et de plusieurs traductions, dont As Teorias Anarquistas (1909) et $A$ Utopia Socialista (1913) ${ }^{1}$. Militant, il collabore à des journaux et à des revues, qu'il dirige parfois. Il s'agit de publications souvent éphémères « de índole diversa, quer propriamente literária [...], quer de engajamento político [...], quer ainda de orientação esotérica espiritualista », précise José Eduardo Reis (2007, p. 95). Aux périodiques cités par ce chercheur et par João Freire, 
il faudrait ajouter la revue Amanhã. Dans cette éphémère revue bimensuelle, qui n'a connu que six numéros, nous avons trouvé trois textes, de nature différente, signés par Ângelo Jorge. Nous les avons découverts par hasard au cours de recherches bibliographiques en vue de la rédaction de l'article sur Irmânia cité plus haut.

En lisant un article sur les revues anarchistes portugaises de João Freire, spécialiste de l'anarchisme au Portugal, une revue retient notre attention en raison de son titre aux consonances messianiques. Il s'agit de la revue Amanhã. Dans son article, João Freire cite à plusieurs reprises Ângelo Jorge mais n'établit aucun lien entre ce dernier et la revue Amanhã. Il mentionne néanmoins un autre périodique bimensuel portant le même titre dont la publication éphémère se limitera à deux numéros en 1922. Il est peu probable qu'Ângelo Jorge ait publié un texte dans cette revue dirigée par l'avocat anarchiste João Evangelista Campos Lima (cf. FREIRE, 2003, p. 160, 164 ; et 2002, p. 272) car il mourut cette année-là, emporté prématurément par, pense-t-on, la tuberculose (cf. BARBOSA, 2003, p. 3). De plus, il ne publiait plus d'ouvrage depuis 1918. N'ayant pas eu accès à cette revue, nous avons consulté par voie électronique la revue $A m a n h \tilde{a}^{2}$ parue entre le 1 er juin et le 15 août 1909 et dirigée par Pinto Quartim et Grácio Ramos. Ce dernier a publié en 1915 O Terrorismo em França de 1891 a 1894, version portugaise d'un livre d'Henri Varennes sur la violence anarchiste (cf. VENTURA, 2000, p. 257, n. 160). Né en 1887 au Brésil où il devra se réfugier à deux reprises pour des raisons politiques, Pinto Quartim fonde $O$ Protesto - Guerra Social en 1908 puis, en 1913, un autre journal anarchiste, Terra Livre. Il dirigera un temps la rédaction du célèbre journal de la Confederação Geral do Trabalho, à savoir A Batalha.

C'est aussi pour contribuer à la diffusion de la pensée libertaire qu'il crée à Lisbonne, avec Grácio Ramos, la revue Amanhã dont la couverture représente trois personnages, l'un pointant le doigt vers le soleil levant. La métaphore de l'aube irradiante véhicule la promesse messianique d'un avenir radieux auquel est promis l'enfant, incarnation d'un futur en construction, qui figure sur l'illustration de la couverture ; le livre que l'un des personnages tient dans sa main symbolise, quant à lui, la raison ${ }^{3}$ sur laquelle se fonde l'espoir d'un monde meilleur. La revue porte d'ailleurs le sous-titre suivant : Revista Popular de Orientação Racional. Dans le premier numéro paru le $1 \mathrm{er}$ juin 1909, les membres de la rédaction se présentent en ces termes dans un

2 Cette revue est consultable sur le site de l'Hemeroteca Digital, à l'adresse suivante : <http://hemerotecadigital.cm-lisboa.pt/index.htm $>$. Nous avons conservé l'orthographe originale dans les citations.

3 Sur le rationalisme auquel sont attachés les anarchistes, voir João Freire (2003, p. 154) et Alain Pessin (1999, p. 102, 112, 113. 
texte intitulé "Ao leitôr " : "Somos os precursores do futuro, os precursores do ámanhã. ». Dans une note précédant ce texte, Grácio Ramos et Pinto Quartim déclarent vouloir faire " obra de educação social » en constituant notamment une "pequena biblioteca racionalista». Le premier article signé par la rédaction et intitulé "Ao leitôr » sert d'introduction programmatique au contenu de la revue :

o que queremos?

Queremos pão, liberdade, ciência e bem-estar para todos os que compõem a grande familia humana. Queremos que a cada individuo assegurado seja o seu mácimo de felicidade. [... ] Queremos, enfim ! que a Vida espluda por toda a parte do glôbo, intensa, plena, ezuberante, esplendida!

- Méras vizões de utopistas - direis. Oh ! não, não ! homens céticos, pessimistas, achacados e impotentes. [...] Não ! o futuro social que o nosso cérebro compreende e o nosso coração ama, é a conclusão de toda a ciência moderna [...].

[...] A ciência é a redentôra da humanidade.

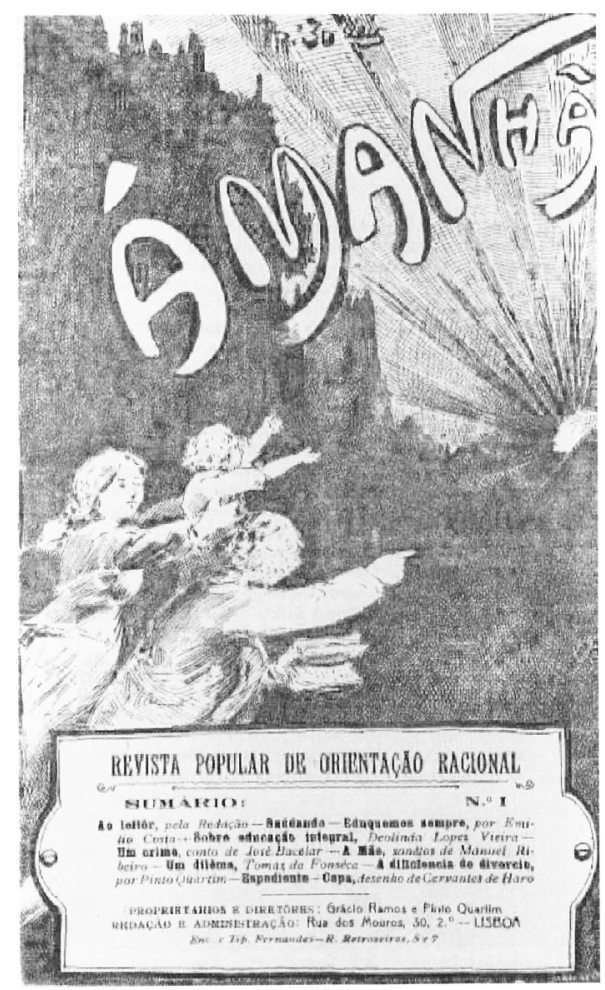

Ce texte se présente donc comme un manifeste dont les auteurs réhabilitent l'utopie dénigrée au siècle précédent par Marx et critiquent ouvertement l'ordre établi : 
"Amanhã » aparece hoje pâra descobrir os êrros que no fundo de cada uma das nossas instituições sociais ezistem ; para espurgar do cérebro dos Homens as ideias falsas legadas por um passado de obscurantismo e de ignominia, e aferradas na mente das nossas gerações pela educação oficial, servil, dogmatica e metafisica.

La monarchie à bout de souffle est à l'évidence dans la ligne de mire de la revue Amanhã qui proclame « a sua adesão a tôdas as iniciativas que tenham por fim a completa emancipação intelectual do pôvo " et qui accueille des articles dérangeants d'Emílio Costa, tourné vers l'éducation et lié alors au mouvement anarcho-syndicaliste ${ }^{4}$, ou d'Ângelo Jorge, libertaire et propagandiste du régime végétarien.

\section{LE SONNET «A FABRICA »}

Dans la livraison du 15 juillet 1909, Ângelo Jorge publie un sonnet en décasyllabes intitulé «A Fabrica » pour dénoncer la condition ouvrière ; il choisit donc à dessein une forme poétique noble pour traiter, en le valorisant, un thème ouvrier :

\section{A Fabrica}

Paro em frente da fabrica maldita

Que se ergue, altiva, a meio duma rua;

$E$ ao vê-la, a alma queda-se contrita

E o coração, de dôr, no peito estua.

Meio-dia na torre. O monstro apita, A lejião dos párias tumultúa.

Um grande borborinho a rua ajita, Eleva-se e no proprio ar flutua.

Oh ! quanta dôr a vida não traduz,

- Penso então - quanto esforço nunca visto,

Vivida assim nas fabricas sem luz!

4 « La question de la participation des anarchistes pour renverser la monarchie a provoqué des discussions et une polémique parmi eux pendant plusieurs années. Emílio Costa, un des plus brillants intellectuels du mouvement libertaire portugais, a publié en 1903 un livre qui avait justement comme titre La République est-elle nécessaire ?, où il met en équation les avantages et les inconvénients d'une telle participation et où il conclut que l'implantation de la république constituerait un progrès pour le peuple portugais et que la contribution des anarchistes ne limiterait en aucun cas leur liberté d'action postérieure.

[...] Ce courant libertaire favorable à la collaboration avec les républicains est resté connu sous le terme d' interventionniste'. " (FREIRE, 2002, p. 46-47 ; voir aussi p. 273). 
Trabalhador escravo, em face disto, Eu julgo mais pezada a tua cruz

Do que a cruz em que foi pregado Cristo!

(JORGE, 1909b, p. 13).

On assiste donc ici à un détournement du sonnet d'un point de vue thématique. Sur le plan formel, le poète s'inspire du sonnet traditionnel en décasyllabes et opte pour une variante des rimes classiques dont le schéma est le suivant : $A B A B A B A B C D C D C D$. Nous avons affaire à des rimes croisées ; Camões, dans les quatrains, privilégiait les rimes embrassées en ABBA, comme dans le célèbre sonnet "Alma minha gentil, que te partiste ». L'auteur de "A Fabrica " choisit plutôt des rimes riches, les termes sur lesquelles elles reposent appartenant souvent à des catégories grammaticales différentes (« rua »/« estua »; « traduz »/« luz»; « disto »/« Cristo »). La rime interne ("vida »/ vivida»; " dor »/« trabalhador») ainsi que l'enjambement ("Que se ergue "; " E o coração») mettent habilement en relief des éléments clés du poème. Les assonances et les allitérations percutantes contribuent également à donner une vision animée et saisissante de la réalité décrite qui est loin d'être réjouissante puisqu'il s'agit de la dure réalité de la vie à l'usine dans un Portugal en cours d'industrialisation lente. Ce n'est donc pas la forme mais le contenu a priori prosaïque de ce sonnet qui est atypique. La forme poétique, noble et exigeante, valorise le contenu dont la valeur que lui reconnaît l'auteur explique le choix du sonnet.

L'espace urbain où se meut le sujet poématique, qui se présente sous les traits d'un promeneur, est dominé par le travail industriel. En effet, l'usine ${ }^{5}$ qui est donnée à voir à travers le regard extérieur de cet observateur solitaire s'élève au beau milieu d'une rue ; le mot « fabrica » figure d'ailleurs à un endroit stratégique du vers, en position médiane. Cette position centrale, dans le vers et dans l'espace, suggère déjà que tout s'organise, dans le poème et dans le monde décrit, autour de ce lieu de travail dont tout dépend. Notons que le sujet poématique est arrêté dans son mouvement au moment même où il passe devant elle. Ainsi, dès le premier vers, l'usine exerce un fort pouvoir sur l'individu. Sa représentation dans l'espace qu'elle domine de sa hauteur ainsi que l'adjectivation péjorative qui lui est associée en font d'entrée de jeu une figure écrasante et castratrice. L'idée de verticalité étouffante est rendue par la forme verbale " se ergue »; l'adjectif déprécia-

5 «Dans l'ensemble, on est passé de 1031 usines, employant 15000 ouvriers en 1822 à 1350 usines et 18000 ouvriers en 1881, jusqu'à 5000 usines et 200000 ouvriers à la veille de la Première Guerre mondiale. " (FREIRE, 2002, p. 26). 
tif " altiva ", placé au milieu du deuxième vers, renforce cette idée tout en contribuant à l'animisation de l'usine présentée comme un lieu maudit, l'adjectif « maldita » étant placé en position finale. La mise en relief s'opère également par le biais de l'accent d'intensité qui intervient sur les deux mots clés du premier vers : "fabrica maldita ». La vue d'un tel bâtiment, faisant partie à l'époque de l'espace urbain, cause une vive impression chez le sujet poématique. De l'objet regardé on passe au sujet regardant, ce passage étant matérialisé par le point-virgule. Le rythme s'accélère et devient nettement irrégulier dans les deux derniers vers du premier quatrain, ce qui traduit le trouble du sujet poématique. Là encore, l'accent rythmique porte sur des termes importants dont certains se trouvent, en outre, au milieu ou à la fin du vers : " alma ", " contrita ", " coração ", " dôr », " estua ». C'est à travers l'agitation intérieure, violente comme le suggère le verbe " estua ", du sujet regardant que l'on percevra émotionnellement la vie à l'usine.

Dans le prolongement de l'animisation négative, la métaphore angoissante $d u$ monstre est convoquée dans le deuxième quatrain pour désigner l'usine dont l'image péjorative continue d'être travaillée par le poète. En effet, les assonances en [i], les allitérations en [t], en [m] et en [b] ainsi qu'un rythme saccadé créent une sensation sonore désagréable. L'usine, d'où sort précipitamment et bruyamment, comme d'une prison, une cohorte d'ouvriers, à l'heure du déjeuner, est ainsi associée à un univers foncièrement dysharmonique et desséchant, d'où l'absence d'adjectifs dans les deux premiers vers. Il s'agit d'un lieu où règne un ordre quasiment militaire, comme le suggère le mot « lejião " dans l'expression "lejião dos párias ". Celle-ci renvoie à un espace collectif générateur de ségrégation socio-économique où les ouvriers, ravalés au rang infamant de parias, sont victimes d'un double rapport de domination et d'exploitation. D'eux, il ne reste qu'une image sonore, une rumeur persistante qui s'élève au-dessus de la ville ; ils sont comme dépersonnalisés, le nom collectif - «a lejião »- les réduisant en quelque sorte à l'anonymat.

Dans le premier tercet, le pathos n'empêche pas chez le sujet poématique une attitude réflexive suggérée par le verbe " Penso ". En voyant les ouvriers sortir de l'usine, le sujet poématique réalise avec émotion la dureté de leur vie, ces individus étant toujours dépersonnalisés. L'interjection de douleur "Oh » qui donne immédiatement le ton de la strophe, le style exclamatif, le vocabulaire de la douleur, l'adverbe exclamatif dans les expressions " quanta dor » et " quanto esforço », le phénomène d'écho entre "vida » et " vivida » ainsi que des termes privatifs exprimant le manque de visibilité ou de lumière contribuent à rendre l'émotion palpable, intense. Par ailleurs, l'adverbe « assim » rend l'évocation plus frappante, plus percutante et favorise l'investissement émotionnel du lecteur. On remarquera, en outre, 
la référence insistante au pénible labeur " nunca visto » des ouvriers. Cette expression, placée en fin de vers, renvoie à l'invisibilité des travailleurs et à l'existence obscure, souterraine qu'ils mènent derrière les hauts murs de l'usine qui apparaît comme un espace concentrationnaire, aliénant et mortifère. L'absence de lumière mise en relief par le point d'exclamation fait d'elle, en somme, un espace régressif de monstruosité et de ténèbres. Par la pensée - «Penso »-, le sujet poématique cherche justement à faire la lumière sur " a vida [...] vivida assim », c'est-à-dire sur la condition ouvrière dans son ensemble, le pluriel généralisant - «nas fabricas » - atténuant quelque peu la subjectivité et conférant une portée universelle à la dénonciation.

Dans le deuxième tercet, non dépourvu de pathétisme et d'empathie manifeste, la dimension réflexive et critique du poème, à laquelle renvoie la forme verbale "Eu julgo ", est accentuée par le recours au démonstratif "isto » dans le syntagme prépositionnel « em face disto " qui produit un effet d'hypotypose et induit un jugement face à une réalité humaine et sociale, laquelle se présente de manière frappante sous les yeux du sujet poématique et du lecteur. L'expression inaugurale "Trabalhador escravo ", qui est d'ailleurs paradoxale pour frapper les esprits, pose comme une évidence l'assimilation du travailleur à l'esclave, l'esclavage salarial étant présenté comme l'esclavage des temps modernes par le biais d'une surprenante analogie. On remarquera que le phénomène d'écho permet de produire une rime particulièrement significative entre "dôr ", terme utilisé dans le premier tercet, et "trabalhador » et que les rimes intérieures sont parfois réduites à la simple répétition d'un mot ("dôr » ou " cruz "), la rime interne jouant son rôle classique de marquage des éléments clés autour desquels se construit le sens du sonnet. Dans une comparaison à valeur superlative, l'ouvrier devient une figure de la souffrance supérieure à celle qu'offre le Christ en croix. Le comparatif "mais " devant le participe passé à valeur d'adjectif, affecté ainsi d'un degré d'intensité, ainsi que les connotations péjoratives de l'épithète " escravo " ou, dans la première strophe, des adjectifs " maldita " et " altiva ", fonctionnent comme des indices de jugement ; ces adjectifs sont d'ailleurs des termes axiologiques. De plus, le nom "cruz ", concret comme l'adjectif " pezada ", est répété pour accentuer le sentiment d'horreur et d'indignation qu'inspire la dureté de la vie à l'usine. Celle-ci est évoquée de manière saisissante à travers l'image obsédante de la croix qui revient comme un leitmotiv lancinant. Dans un crescendo émotionnel, le sujet poématique jette donc sur l'ouvrier portant sa croix un regard plein de compassion, compassion dont l'intensité est traduite par le point d'exclamation qui vient clore la dernière strophe du poème. 
Ainsi, la vision du monde ouvrier véhiculée par ce sonnet composé à l'aube du XXe siècle est diamétralement opposée à celle que véhiculait au siècle précédent la littérature bourgeoise travaillée par l'utopie de l'harmonie des classes. Nous sommes loin, en effet, du récit de la réussite et de la performance individuelles pratiqué, par exemple, par le dramaturge Ernesto Biester qui, au XIX ${ }^{e}$ siècle, aime à mettre en scène, dans ses pièces, le bon ouvrier qui parvient à devenir un patron exemplaire (cf. PEREIRA, 2011, p. 83-115). Cette vision aliénante et idyllique du monde ouvrier vise à légitimer l'ordre bourgeois capitaliste attaché au mythe du self made man. On distingue nettement deux parties dans ce sonnet d'Ângelo Jorge qui fait appel à l'émotion et, pour que celle-ci ne tourne pas à vide, au jugement critique, lequel s'affirme dans le dernier tercet, d'où l'emploi de la forme verbale " Eu julgo ». Tout d'abord, le poète nous livre dans les deux quatrains la description d'une réalité extérieure : l'usine hurlante et ses ouvriers en milieu urbain. Face à ce spectacle extérieur, il s'opère alors dans les deux dernières strophes du sonnet une prise de conscience, aiguë et sensible, de la vie souterraine et cependant réelle des ouvriers, cachée derrière le mur d'enceinte de l'usine, emblème de la condition ouvrière. Cette deuxième phase est annoncée par la forme verbale "Penso então "; on passe très vite de la réflexion au jugement, comme l'indique le recours à la forme verbale " $\mathrm{Eu}$ julgo ». Mais, dans ce sonnet, la réflexion va de pair avec l'émotion qui va crescendo et le lecteur qui devient un observateur, un spectateur comme le " je " du poème auquel il s'identifie et qui oscille entre une posture lyrique et une posture réflexive et critique, est amené à compatir lorsqu'il réalise la dureté de la condition ouvrière qui est ici dénoncée avec force.

Dans ce poème, on remarquera qu'Ângelo Jorge conjugue anarchisme et christianisme, transformant dans le dernier tercet le travailleur en une figure christique de la souffrance : «Trabalhador escravo, em face disto, / Eu julgo mais pezada a tua cruz / Do que a cruz em que foi pregado Cristo ! ». A ce propos, José Eduardo Reis (2004, p. 16) note dans son introduction que " para Ângelo Jorge, mesmo o da fase de simpatia pela teoria materialista da história e da teoria social anarquista, o modelo ou 'símbolo eterno' do utopista [é] representado pela figura de Cristo, 'o meigo sonhador, o grandioso revolucionário [...]' ». En définitive, il n’y a pas d'homogénéité ou d'unité de pensée dans la sphère anarchiste et encore moins chez Ângelo Jorge. Ainsi, des anarchistes passent dans le camp des républicains peu avant l'avènement de la première République (cf. VENTURA, 2000, p. 212) et, d'après Manuel Braga da Cruz (2003, p. 48), au début du XXe siècle, "Muitos foram os operários de matriz anarquista que, em Portugal e na Europa, ingressaram nos movimentos católicos. ". Les débuts de la première République ont été particulièrement mouvementés en raison des nombreuses grèves, souvent 
spontanées, qui agitaient le pays (cf. VENTURA, 2000, p. 214-215) et que certains anarchistes condamnaient. D'après António Ventura (2000, p. 221), Ângelo Jorge " criticava, em 1911, os grevistas seus antigos companheiros " ; deux ans plus tôt, il avait cependant plaidé la cause ouvrière dans le sonnet publié dans la revue Amanhã, que nous venons de commenter.

Dans les deux autres textes parus en 1909 dans cette même revue, Ângelo Jorge expose deux principes importants de l'anarchisme. En effet, dans le numéro 2 du 15 juin 1909 et dans le numéro 6 du 15 août 1909, il publie deux articles à caractère idéologique, intitulés respectivement « $\mathrm{O}$ amôr sécsual » et «A fórmula autoritaria ».

3. "O AMÔR SÉCSUAL » OU L'AMOUR LIBRE

Les anarchistes militaient pour l'émancipation de la femme et pour l'amour libre (cf. FREIRE, 2002, p. 274 et suiv.), que Giovanni Rossi a théorisé puis mis en pratique dans la colonie Cecília et que le libertaire Ângelo Jorge a défendu également. Au sujet de l'amour libre, voici ce qu'écrit ce dernier dans " $O$ amôr sécsual " : " Para ser puro e verdadeiro, haveria de sêr livre. " (JORGE, 1909a, p. 5). Ângelo Jorge ne sera pas le seul à traiter de cette question puisqu'un article intitulé précisément "Amor livre " paraîtra en 1913 dans l'Almanaque de A Aurora (cf. FREIRE, 2003, p. 177). Dans le numéro 6 de la revue Amanhã, Coriolano Leite $(1909$, p. 10) publie un poème provocateur composé de deux sonnets en alexandrins qui porte le même titre et commence par ces vers : "Virjens : erguei o olhar que as sombras do convento / acostumou a andar cerrado pára a luz. Deixai um instante só os êstasis da cruz, / e enchei-vos deste sôl que brilha turbulento. ». Le premier sonnet s'achève par ces vers : « Vinde gozar a vida em toda a plenitude / e não faneis assim a vossa juventude / com sonhos infantis duma banal pureza. " (LEITE, 1909, p. 10). Le message est sans ambiguïté une fois qu'on a lu la dernière strophe du deuxième sonnet où le sujet poématique apostrophe les hommes : " E vós, homens do amor, e vós que as desejais, / arrancai-lhes da fronte as c'róas virjinais, / beijai-as livremente á grande luz do dia. » (LEITE, 1909, p. 10). Cette invitation à l'amour libre publiée sous une monarchie catholique vacillant sur ses fondements se veut blasphématoire. L'appel à la jouissance dans ce texte, comme dans d'autres, explique l'association dénigrante libertaire/libertin. Voici ce qu'écrit Alain Pessin (1999, p. 112), qui interroge la relation existant entre « libertarisme et libertinage» :

Soumis constamment au reproche de l'inévitable licence qui proviendrait d'une liberté effrénée, les anarchistes ont été soucieux de prendre distance par rapport aux abîmes du désir. Même chez les 
individualistes les plus passionnés [...], la liberté ne peut s'engager sur la pente de tels excès [...]. [...] il existe pour lui [Stirner] au moins deux types d'hommes non-libres : d'une part ceux qui dépendent d'une croyance, et d'autre part ceux qui dépendent de leurs impulsions naturelles, catégorie à laquelle le libertin semble voué [...]. Les individualistes et les collectivistes libertaires [comme Giovanni Rossi] se rejoignent donc ici pour considérer que ce n'est pas une limite à la liberté, mais au contraire une condition indispensable à son existence [...] que de s'établir dans un processus auto-régulateur.

Les anarchistes sont divisés quant à la conception de ce processus auto-régulateur :

Ce sera chez les individualistes la raison humaine qui, le plus souvent, remplira ce rôle [...], ce sera chez les collectivistes la reconnaissance [...] d'une régulation sociale, reconnaissance d'une autorité dans le fait social, acceptée dans la mesure où il ne s'agit pas d'une autorité séparée [étatique ou religieuse] mais de l'autorité du social lui-même. [...]

Aussi n'est-ce pas dans le culte de la jouissance que nous pourrions reconnaître un libertinage anarchiste, mais bien davantage en comprenant ce dernier comme art de la rupture [...]. [...] Si cette révolte s'accomplit dans une manière de libertinage, celui-ci n'est donc pas à lire dans l'étroit point de vue du séducteur, mais bien plus dans son complément, celui de la nécessité de savoir refuser les tentations adverses, s'arracher aux séductions de l'ordre, de l'établissement, de la sécurité [...]. (PESSIN, 1999, p. 112-114).

Dans la longue et habile entrée en matière de son article de deux pages, Ângelo Jorge (1909a, p. 4) questionne tout d'abord l'idéal amoureux éthéré avant de s'en prendre sans ambages à l'ordre moral bourgeois qui n'empêche guère l'individu de tomber dans les « abîmes du désir ». "Os espíritos superiores que vivem na serena contemplação interior das formas mais belas da Vida [...] pássam na terra [...] tristes [...]. [...] // Espíritos de luz, almas d'amôr, gástam-se na constante e dolorosa idealização dum côrpo perfeito de mulher, ardem num escésso de vida moral [...]. ». Pour mieux ménager la chute, l'auteur semble au début de son texte se ranger derrière les idéalistes de l'amour, toujours insatisfaits. La critique se fait jour : "Ai dos tristes ! A sua concéção da Belêza e do Amôr é alta de mais pâra a baixeza dos tempos que decorrem, e a sua vida psíquica, intensa em demazia perante sêres em que as funções dijestivas são as supremas funções vitais [...]. " (JORGE, 1909a, p. 4). Ângelo Jorge (1909a, p. 4-5) dénonce alors clairement le matérialisme et la morale en trompe-l'œil de la société bourgeoise qui ne fait guère de place aux idéalistes : 
Pedem amizade e dão-lhes a intriga tôrpe, o Deve e Haver utilitarista ; suplicam amôr e oferécem-lhes a prostituïção. E enquanto sêres sem nobreza nem elevação anímica se refocilam no lôdo dos prazêres venais, êles passeiam [...] a grande tortura que nêles bróta da insatisfação dum ideal d'amôr que a época não comporta.

Ah ! o amôr sécsual, pâra êles, deveria sêr alguma cousa bem diversa do que é agora !

Ângelo Jorge (1909a, p. 5) laisse les idéalistes à leur tristesse et amène subtilement le sujet par le biais de l'idéal amoureux éthéré vis-à-vis duquel il prend désormais ses distances :

Para ser puro e verdadeiro, haveria de sêr livre. Livre de preconceitos que infamam, de regras que tiranizam e desvirtuam.

A mulher, colocada num plano economico igual ao do homem, tendo socialmente os mesmos direitos, não por igualdade de deveres, por identidade de missões a cumprir, mas sim pela sua simples condição de sêr humâno, com logar marcado e assente no banquete da Vida.

L'auteur penche donc nettement pour un idéal amoureux qui revendique la liberté absolue en amour et invite à ne pas confondre la prostitution et l'infidélité conjugale, qui sont autant de signes de décadence du monde bourgeois, avec l'amour libre, source de régénération morale. Dans un plaidoyer pour l'émancipation de la femme qui ne doit pas être réduite à la femme-objet, à un objet sexuel ou à un simple agent de (re)production, il oppose " a mulher-boneca, a mulher-escrava, a mulher-máquina de gôzo " à la «mulher dignificada e livre », c'est-à-dire à la " mulher dona do seu corpo e dona da sua alma" (JORGE, 1909a, p. 5). La société bourgeoise a fait de la première une " mulher sem personalidade que um pai, um irmão ou um amante tiraniza ", tandis que la société libertaire ferait de la seconde une "mulher vivendo a mesma vida moral e mental do homem, e contribuindo com o seu esfôrço consciente e positivo pâra o progredimento da sociedade, pâra o embelezamento da Vida " (JORGE, 1909a, p. 5). Il faut soustraire la femme au principe hypocrite de l'honneur et à la tyrannie de la virginité et la reconnaître comme un sujet désirant et pas seulement comme un objet désiré :

A mulher libertada do preconceito estupido da Honra, que a fórça a imolar a sua parte de gôzo e de bem-estar no altar da Virgindade ; a mulher libertada das aperreantes condições economicas que a obrigam a entregar-se á prostituição, êsse cancro que róe a sociedade moderna, condenáda á morte sem remedio. (JORGE, 1909a, p. 5).

Ângelo Jorge (1909a, p. 5) s'attaque également à l'ordre familial patriarcal et bourgeois qui constitue l'obstacle majeur à l'amour libre : «O 
filho é pertença de seus pais, como a mulher é pertença de seu esposo : o sentimento da propriedade estendendo-se dos objétos ás pessoas. // [...]//O respeito ezigido pelos pais aos filhos é absurdo e caricato - conseqüencia do principio d'autoridade [...]. ». Les rapports familiaux traditionnels sont ainsi dénoncés comme des rapports de domination et de soumission au chef de famille, raison pour laquelle Ângelo Jorge (1909a, p. 6) parle de « vassalagem dos pequenos pâra com os grandes ". En bonne logique, il prône de manière radicale la destruction de la famille bourgeoise : "Conseqüentemente, a Familia legal, baze de toda a discordia humana, destruída para sempre. " (JORGE, 1909a, p. 5). Pour les anarchistes, la famille doit être « livre na sua constituição, baseada no amor e na responsabilidade ", comme le rappelle João Freire (2003, p. 154).

$\mathrm{Au}$ mariage bourgeois reposant sur un contrat, Ângelo Jorge (1909a, p. 5) propose de substituer l'union libre fondée sur le désir et le libre consentement des partenaires : "Os dois sécsos entregando-se livremente, na plenitude do seu vigôr e do seu desejo, e amando-se livremente, por tempo indeterminado, sem regras pré-estabelecidas, sem sujeição à vontade de terceiro, escutando tão só [...] a voz íntima do coração, os dítámes rétos da vontade. ». Et Ângelo Jorge (1909a, p. 6) de conclure : «E, d'essárte, o amôr se nobilitaria e engrandeceria, se tornaria, de contráto mercenário e puramente carnal que é, em aliança pura e digna de duas almas e de dois corações, de dois espíritos e de dois corpos, aliança em que iriam implicitos o rejuvenescimento da ráça e a belêza da Vida. ". Pour atteindre leur idéal amoureux, aspiration légitime, les esprits éthérés devraient, par conséquent, commencer par promouvoir l'amour libre : «E, então, os espiritos superiores que vivem na serena contemplação interior das fórmas mais bélas da Ezistencia [...], $[\ldots]$ que agora sofrem e choram - ái dos tristes ! [...] - seriam felizes [...], vendo materializar-se num corpo puro de mulher [...] o seu inatinjivel ideal de belêza e d'Amôr !" (JORGE, 1909a, p. 6). L'amour libre s'impose comme une évidence à la fin de l'article qui séduit par sa force démonstrative.

Derrière le vitalisme et la régénération morale se profile le mythe de l'homme nouveau anarchiste ; le vitalisme caractéristique de l'anarchisme ${ }^{6}$ ainsi que la régénération morale impliquent par ailleurs une rupture frontale avec la morale établie. Ainsi, ce n'est pas au nom du libertinage, mais au nom d'une éthique fondée sur le désir qui n'obéit qu'à lui-même et s'affranchit de toute autorité extérieure - une éthique " assente na consciência individual, no hedonismo e no respeito pela natureza » (FREIRE, 2003, p. 154) - qu'Ângelo

6 «La place attribuée à la subjectivité, à l'individu, aux éléments humains tels que la volonté, le désir ou le plaisir ont conduit certains à identifier l'anarchisme à la philosophie vitaliste. "(FREIRE, 2003, p. 188). 
Jorge fait la promotion de l'amour libre, lequel participe d'une révolution morale qui reste à mener pour construire la société idéale libertaire : " [...] la femme sera libre le jour où l'homme le sera. Pour l'un comme l'autre, seule la Révolution sociale pourra leur apporter la liberté absolue [...]. Celle-ci sera l'heure radieuse de l'Anarchie. ", écrivait un anarchiste portugais en 1908 (cf. FREIRE, 2002, p. 275).

\section{4. «A FóRmUla AUTORITARIA » OU LA DESTRUCTION DE L’ÉTAT}

Le rejet de la loi du Père, dont l'autorité paternelle, disqualifiée dans le texte présenté ci-dessus, est la manifestation la plus évidente, conduit tout naturellement au refus de l'État dont il est question dans "A fórmula autoritaria ». Dans cet article, qui s'étend sur cinq pages et sur lequel s'ouvre le sixième numéro de la revue Amanhã, Ângelo Jorge (1909c, p. 1) commence par remettre en cause le darwinisme social qui sert à justifier les inégalités sociales : "O struggle-for-life, interpretado por um falso cientismo pretensamente abordado em Darwin, é um erro. ». Puis il poursuit : "A luta vital, em principio, é a constante apreciação das forças da Natureza pelos homens pára determinação duma lojica e racional evolução especifica, e não a vitoria fatal e absurda dos fortes contra os fracos [...]. " (JORGE, 1909c, p. 1). Il conclut alors : "A luta das espécies não é, de forma alguma, a guerra de irmão contra irmão. Não se devora o lobo ao proprio lobo. » (JORGE, 1909c, p. 1). L'homme est un loup pour l'homme, comme l'a dit Plaute avant Hobbes, ce qui signifie qu'il ne sait pas, ou ne sait plus, reconnaître son semblable. Par « luta moderna do forte contra o fraco ", il faut entendre la lutte " do capital contra o salario, da autoridade contra o governado " et le " triunfo da astucia, da maldade, da hipocrisia e do crime" (JORGE, 1909c, p. 1, 2).

Sur le plan politique, la loi du plus fort se traduit par la mise en place d'un au service non des faibles mais des puissants : «Certo é que essa terrificante luta do forte contra o fraco [...] se fez a lei intrinseca, essencial da Vida. A fórmula autoritaria foi criada. $\mathrm{E}$ a breve trecho o Estado a concretizava numa mostruosa abstração. " (JORGE, 1909c, p. 2). L'anarchiste ne reconnaît aucune autorité étrangère à l'individu, qu'elle soit économique, comme le patronat, religieuse - les « caricatas fantasmagorias da liturjia católica » et la « desfeita lenda da Criação segundo a Biblia » (JORGE, 1909c, p. 1, 2) sont d'ailleurs évoquées - ou politique, comme l'État au sujet duquel anarchistes et communistes s'affrontent. Comme le rappelle António Joaquim de Sousa (1995, p. 14), Kropotkine, auquel se référaient les anarchistes portugais (cf. MESQUITA, 2006, p. 525, 527, 537), disait que la société est traversée par deux tendances antagonistes, "uma corrente autoritária e uma corrente 
libertária "; le titre de l'article qui nous occupe renvoie au courant autoritaire. Le libertaire Ângelo Jorge (1909c, p. 2), qui se fait idéologue, critique au passage la théorie marxiste de l'État : "Só será lejítimo o Estado pâra eliminar o proprio Estado. É o caso do socialismo " - proclama-se // Não, não. $O$ Estado não é nunca lejítimo. $O$ Estado não cura nunca de eliminar $o$ proprio Estado. Não se devora o monstro a si proprio. ». De là la distinction entre le socialisme libertaire et le socialisme autoritaire, dit aussi « scientifique », qui n'est autre qu'un socialisme d'État (SOUSA, 1995, p. 6, 12, 13, 14) ; António Ventura $(2000, \text { p. } 79)^{7}$ fait d'ailleurs allusion au « debate em torno da divisão do movimento socialista em 'autoritários' e 'libertários ". Développant sa conception anarchiste de l'État, Ângelo Jorge (1909c, p. 3) ajoute : "Estado que se destroe a si proprio é utopia de injenuos. ". C'est pourquoi il rejette le socialisme parlementaire, l'État socialiste étant la dernière étape avant la disparition de l'État censée inaugurer l'ère communiste ${ }^{8}$ :

o socialismo parlamentar, que significa, tão só, um élo a mais, o ultimo porventura, da cadeia evolutiva-regressiva do Estado deve, portanto, a sua ezistencia como teoria e como modo d'ação revolucionaria, ao mesmo fenómeno a que os vários principios politicos devem a sua formação : - á incompleta educação filosofica e social das classes trabalhadoras e oprimidas. (JORGE, 1909c, p. 4).

Le discours d'Ângelo Jorge fait écho à celui de Bakounine qui reprochait à Marx « la naïveté de croire - ou de faire croire - à une modification de nature possible pour l'Etat, pour peu que son personnel vienne à changer "(PESSIN, 1999, p. 98). Alain Pessin (1999, p. 98-99) explique la position anti-étatique de Bakounine qui était aussi populaire auprès des anarchistes portugais que Kropotkine (cf. VENTURA, 2000, p. 79, 85, 98, 139, 142, 203) :

Tout d'abord, l'Etat, pense-t-il, transcende les modalités particulières de la domination. [...] sa nature profonde ne se modifie pas en fonction de la classe qu'il sert et des foules qu'il dessert. [...] L'Etat n'est donc pas cette superstructure fragile et fluctuante

7 Voir également António Pedro Mesquita (2006, p. 529).

8 "Le socialisme n'est pas encore le communisme.

Il comporte un État, un appareil d'État, donc lui aussi une bureaucratie, un appareil répressif, un appareil juridique. [...]

Sous l'angle politique, le communisme se définit par la liquidation définitive de ces survivances, de ces prolongements. Pour beaucoup de gens qui ignorent cet axiome de la pensée marxiste, il faut dire et répéter que l'expression 'État marxiste' est dépourvue de sens. En effet, le communisme est caractérisé par la suppression de l'État, par son dépassement.

Au cours de la période socialiste, transition vers le communisme, l'État se transforme progressivement. [...] Alors l'État dépérit comme tel [...].

Cette disparition de l'État annonce la société communiste. " (LEFEBVRE, 2006, p. 100- 
qu'ont voulu voir en lui les marxistes, il est l'instance autonome de la domination, d'autant moins fragile [...] que sa tendance, que la pente naturelle de tout pouvoir est de s'étendre sans cesse, de gagner peu à peu à son règne tous les aspects de la vie individuelle et collective.

Le propagandiste portugais de l'anti-étatisme s'inquiète, comme nous l'avons lu plus haut, de l'« incompleta educação filosofica e social das classes trabalhadoras e oprimidas "; " razão " et " livre-ezame " (JORGE, 1909c, p. 2) sont les deux principes qui devraient guider les éducateurs. Pourtant, même le courant le plus progressiste a encore beaucoup de mal à renoncer, dans la pratique, à la "formule autoritaire ", ce qui l'empêche, naturellement, de former l'homme libre : «E o que mais fortemente nos espanta é que as proprias escolas que inscreveram em seus programas a abolição do Estado e se dizem plenamente libertadas da superstição política, se revelem por igual aferradas, no seu modo d'ação pratica, a essa antiquada fórmula opressiva. " (JORGE, 1909c, p. 2). Notons qu'il existait une école tournée vers la pédagogie libertaire, fondée en 1908 à Irmânia et appelée " Escola Livre da Irmânia » (ANONYME, 2010) ; les écoles dites " libres » ont vu le jour au Portugal à partir de 1906 (cf. FREIRE, 2002, p. 197, 272, 273).

Ângelo Jorge (1909c, p. 3) définit ainsi l'État : « [...] o Estado representa a fórmula opressiva das minorias sobre as maiorias [...]. » ; "Estado quer dizer minoria organizada. [...] Estado e opressão sinonimizam-se. ". L'État sert donc les intérêts du groupe qui détient le pouvoir. Celui-ci, même s'il est conquis démocratiquement, est toujours le produit de la " formule autoritaire " puisqu'il est confisqué d'une certaine manière au peuple vu que ce dernier l'exerce par procuration, ce qui revient à dire qu'il ne l'exerce pas réellement. On le sait, les anarchistes défendent « o conceito libertário de uma comunidade autogovernada, descentralizada e federalizada " (SOUSA, 1995, p. 13) ${ }^{9}$; ils aspirent donc, d'après João Freire (2003, p. 154), à « uma sociedade livre, sem Estado, ou seja com uma socialização e disseminação do poder político por todo o corpo social ». L'auteur de «A fórmula autoritaria » continue d'exposer la conception anarchiste de l'État et du pouvoir en s'appuyant de nouveau sur la théorie de l'évolution :

O Estado sofre, como todos os outros organismos sociais, a influencia permanente da evolução. Evolução que nele é regressiva.

[...] A passagem do absolutismo pâra o constitucionalismo, o presidencialismo, o sufrajio universal, a queda do principio do poder hereditario dos reis, etc., são já as modificações regressivas dos primitivos poderes do Estado. (JORGE, 1909c, p. 3). 
Ainsi Ângelo Jorge voit-il dans les transformations de l'État un évolutionnisme à rebours ; il en veut pour preuve le maintien de la propriété privée ${ }^{10}$, quelle que soit la "formule autoritaire " adoptée : "Mas tais modificações [...] não trazem comsigo decisivos melhoramentos á estrutura social, cujo erro d'orijem consiste no principio iniquo da proprièdáde priváda que atravez delas sempre subsiste. » (JORGE, 1909c, p. 3-4) ; pour les anarchistes, l'organisation sociale doit reposer sur des bases égalitaires. L'État se métamorphose donc au gré des changements de régime mais ne disparaît pas : " Nem tais modificações deixam supor, tão pouco, que a fórmula autoritaria traga a morte dentro dêla propria. " (JORGE, 1909c, p. 4). Ângelo Jorge (1909c, p. 4) associe ensuite l'État à un principe statique alors que la vie est un principe dynamique : " [...] o Estado é a inércia das formas criadas quando o movimento é a lei fundamental da Vida [...]. ". Alain Pessin (1999, p. 102-103) a montré que les libertaires sont très attentifs à la dynamique de la vie individuelle et collective, raison pour laquelle «les écrits anarchistes qui permettraient de concrétiser l'image de la société future sont extrêmement rares et succincts » :

Ce serait, pense-t-on, céder à nouveau à l'autoritarisme que de proposer [...] des modèles pour la vie. [...] les libertaires se refusent à se prévaloir d'une intelligence de la vie sociale qui entreprend de se systématiser, pour induire les formes à venir de l'existence collective. [...] la rêverie libertaire [...], sensible aux possibles tyrannies de l'abstraction, préfère attendre le futur, s'en remettre - avec joie et confiance, souligne Bakounine - à l'instinct des foules, libertaire et solidaire, et à leur imagination, pour la construction des mondes à venir ; s'en remettre en confiance à la vie, " toute fugitive et passagère, mais aussi toute palpitante de réalité et d'individualité, se sensibilité, de souffrances, de joies, d'aspirations, de besoins et de passions " $[\ldots]^{11}$

L'État, en tant que « monstruosa abstração » (JORGE, 1909c, p. 2), est la principale des abstractions battues en brèche par les libertaires. Si les masses étaient suffisamment formées politiquement, elles devraient profiter de l'avènement de la République pour éviter de passer par la quatrième phase de l'évolution négative de l'État qui correspond à l'instauration du " socialismo parlamentar », lequel constitue, d'après Ângelo Jorge (1909c, p.

10 Sur la condamnation de la propriété privée par les anarchistes, voir António Pedro Mesquita (2006, p. 527-528).

11 Sur l'anti-dogmatisme libertaire, voir António Joaquim de Sousa (1995, p. 12). 
4), le dernier maillon " da cadeia evolutiva-regressiva do Estado », comme nous l'avons vu plus haut : «[...] se as massas trabalhadoras [...] tivessem já, por uma perfeita educação filosofica, conciencia plena da sua missão, o termo da evolução regressiva do Estado seria já a Republica burgueza dentro dela tendo de se efetuar o largo movimento revolucionario que nos levará á perfeita e autentica Liberdade. ". Ângelo Jorge anticipe sur les événements, annonçant la fin prochaine de la monarchie constitutionnelle. Mais le régime républicain ne sera pas le dernier stade de l'évolution de l'État; il faudra en passer par le socialisme d'État et la violence révolutionnaire donnée comme régénératrice :

Certo que o advento do $4 .^{\circ}$ Estado, já agora inevitavel, será bem o termo da evolução regressiva que se opera ha seculos quanto á concretisação abstrata da chamada fórmula autoritaria. Mas menos verdade não é que a abolição total do Estado só se terá efetuado quando as multidões, desiludidas, pela pratica, das teorias do socialismo parlamentar, tiverem imposto pela violencia a anarquia pura, franca e decisiva. (JORGE, 1909c, p. 4).

Le socialisme d'État est présenté par Ângelo Jorge, nous l'avons vu, comme une chimère d'utopistes naïfs et l'État comme une "superstição politica » (JORGE, 1909c, p. 2), un mythe politique ; aussi doit-il être détruit, conformément au credo anarchiste : " a violencia cura-se com a propria violencia ", assène Ângelo Jorge (1909c, p. 3). Puis ce dernier poursuit : "Não é o Estado eliminando o propio [sic] Estado. É o Estado cedendo terreno ante a ação direta e revolucionaria das multidões. " (JORGE, 1909c, p. 4). Seule l'action directe, violente des masses pourra venir à bout de l'État, la transformation sociale ne s'obtenant que par la voie révolutionnaire : "Abaixo o Estado ! - gritamos nós tambem, [...] porque o Estado é o crime, a opressão, a tirania, o absurdo, a iniquidade !" (JORGE, 1909c, p. 4). S'agissant de l'appel à la violence aux accents bakouniniens ${ }^{12}$ contenu dans cet article, on citera une fois encore Alain Pessin $\left(1999\right.$, p. 117, 119) ${ }^{13}$ qui commente les positions de Bakounine, de Kropotkine et de Malatesta à ce sujet :

L'homme anarchiste est constamment partagé entre une nostalgie rebelle, rêverie aventureuse où l'acte de violence fonderait sa différence en l'excluant de l'ensemble social, donc une image à coloration héroïque, et une représentation mystique de lui-même $[\ldots]$, rêverie $[. .$.$] de repos dans la paix sociale et l'harmonie com-$ 
munautaire. Aussi la réprobation de la violence est-elle toujours présente $[\ldots]$.

[...] Il reste que cette réprobation initiale, au moins implicite chez tous les libertaires, se trouve confrontée à la nécessité pratique d'imposer un principe social nouveau, ce qui amène de proche en proche à concevoir la violence comme fatale, imposée au niveau le plus immédiat par la résistance bourgeoise [...].

Errico Malatesta préconisait « la règle morale et politique de la violence minimale' ", comme le rappelle João Freire (2002, p. 263) ${ }^{14}$ qui ajoute : "Lillégalisme et la violence quasi indiscriminée sont des formes d'action minoritaires, pour lesquelles seuls quelques militants étaient disponibles [...]" (FREIRE, 2002, p. 268). Dans «A fórmula autoritaria », Ângelo Jorge se prononce clairement, notons-le, pour le recours à la violence politique dans une situation révolutionnaire. Il s'agit d'une violence apocalyptique et donc régénératrice puisqu'elle engendrera " a anarquia pura ", autrement dit un monde nouveau ${ }^{15}$ : " la passion de la destruction est en même temps une volupté créatrice » (cf. PESSIN, 1999, p. 145), écrivait Bakounine.

On remarquera enfin la conclusion messianique de l'article d'Ângelo Jorge (1909c, p. 4-5) : « Em nome da verdade, em nome da justiça, em nome da igualdade : que o Estado cáia ! E para que o Estado cáia, [...] olhemos ao lonje, muito ao lonje, pâra o alto, muito pâra o alto, na noute profunda dos tempos do Futuro onde a Liberdade plena e autentica refulje em todo o seu esplendôr. ". La vérité, la justice, l'égalité et la liberté, valeur suprême pour un libertaire, apparaissent donc inscrites dans un temps messianique, utopique. On remarquera ici la référence à la nuit qui donnera naissance à des temps nouveaux, cette référence relevant d'un symbolisme apocalyptique et prophétique évident ${ }^{16}$. L'image de l'aurore que l'on trouve dans l'illustration de la couverture de la revue Amanhã va de pair avec celle de la nuit ${ }^{17}$.

\section{Conclusion}

Les trois textes d'Ângelo Jorge parus dans cette revue anarchiste - un sonnet à thématique ouvrière et deux articles de propagande - sont restés, à notre connaissance, ignorés des spécialistes. Leur publication en 1909 correspond tout à fait à la phase anarchiste d'Ângelo Jorge décrite par

14 Voir aussi Alain Pessin (1999, p. 118).

15 Sur l'idée de destruction fondatrice à l'œuvre dans l'idéologie anarchiste, entre autres, voir Alain Pessin (1999, p. 142, 144, 154). Voir également John Gray (2008, p. 44, 99, 194). 16 "La nuit symbolise le temps des gestations, des germinations, des conspirations, qui vont éclater au grand jour en manifestations de vie. " (CHEVALIER et GHEERBRANT, 1982, p. 682).

17 «...] la nuit n'est que nécessaire propédeutique du jour, promesses [sic] indubitable de l'aurore. " (DURAND, 1992, p. 224 ; voir aussi p. 99). 
José Eduardo Reis ${ }^{18}$. Cette année-là, l'auteur d'Irmânia travaille, en effet, de manière active à la diffusion des idées anarchistes, comme le montre joão Freire (2013, p. 165) qui fait observer que la propagande est l'arme principale des anarchistes (cf. FREIRE, 2002, p. 155, 268). Le poème d'Ângelo Jorge sur la condition ouvrière ressortit au " poema de fundo social » pratiqué par les anarchistes : "Outro traço comum é a preocupação da leitura literária, do conto ou do poema de fundo social, que inspire à revolta ou à tomada de consciência. » (FREIRE, 2003, p. 176). Ce type de littérature produite par les libertaires remplissait la fonction que Kropotkine assignait à l'art :

No contexto da chamada arte social, definida por Kropotkine, entre outros, a estética anarquista baseava-se num postulado essencial : a arte é um fenómeno social, devendo, assim, a obra criadora exprimir as tendências e aspirações da colectividade. Isto implicava uma fundamentação ético-social da arte que exigia o reconhecimento, por parte das classes trabalhadoras, da sua luta social e dos seus ideais de criação artística. Por outro lado, a estética anarquista valorizava o acto artístico espontâneo como uma forma de acção social, apreciando-se mais a arte de situação do que a conseguida tecnicamente. Pretendia-se dessa forma desmistificar a obra de arte laureada [...]. Desta estética ressaltava a noção colectiva, popular, da arte, na medida em que ela pertencia ao povo [...]. A obra de arte convertia-se, desse modo, numa arma [...]. (VENTURA, 2000, p. 156).

Comme nous l'avons vu, Ângelo Jorge n'a pas négligé la forme lorsqu'il a composé le sonnet intitulé «A Fabrica ». Les deux autres textes qu'il a fait paraître dans la revue Amanhã frappent par leur audace et leur radicalité. Ce discours politique radical avait de quoi inquiéter la monarchie dont Ângelo Jorge prédit d'ailleurs la disparition imminente, faisant ainsi preuve de courage à une époque où le régime monarchique aux abois maintenait en prison ou en déportation des anarchistes (cf. FREIRE, 2002, p. 40-42).

Les trois textes d'Ângelo Jorge que nous avons présentés ici offrent l'intérêt d'aborder les principaux sujets qui mobilisaient les anarchistes : l'amour libre, la condition de la femme, la famille, l'autorité, la condition ouvrière, le capital, le salariat, l'État, la propriété privée, l'organisation sociale, le pouvoir, la violence politique, l'action directe, l'éducation, la religion, la vie comme principe dynamique.

18 «Primeiramente, manifestando-se no plano político, orienta-se essa obra [d’Ângelo Jorge] para a defesa dos direitos e para a libertação do 'povo trabalhador'. É a fase compreendida, grosso modo, entre 1901 e 1910, que corresponde à sua actividade de publicista empenhado em difundir o ideal libertário em periódicos de duração efémera [...] e de tradutor de textos de conteúdo doutrinário. É também a fase em que publica os seus primeiros livros de poemas de desencanto íntimo e de denúncia social [...]. " (REIS, 2004, p. 11-12). 


\section{RÉFÉRENCES BIBLIOGRAPHIQUES}

ANONYME. Escola livre da Irmânia-Marmeleira (Mortágua). República em Mortágua, [En ligne]. http://republicamortagua.blogspot.fr/2010/03/as-escolas-livres-no-concelho-de.html, [15 janv. 2013], 16 mars 2010.

BARBOSA, Iza Luso. O Utopista portuense Ângelo Jorge : Subsídios para a sua biografia. E-Topia : Revista Electrónica de Estudos sobre a Utopia, n ${ }^{\circ}$ 5, [En ligne], http:// http://ler. letras.up.pt/uploads/ficheiros/1648.pdf, [7 janv. 2013], p. 1-5, 2006.

CHEVALIER, Jean et GHEERBRANT, Alain. Dictionnaire des symboles. Ed. rev. et augm. Paris: Robert Laffont « Bouquins »/Jupiter, 1982.

DURAND, Gilbert. Les structures anthropologiques de l'imaginaire. Paris: Dunod “ Psycho-sup ", 1992.

CRUZ, Manuel Braga da. Entre nacionalismo e democracia cristã. In : REIS, António, LOURENÇO, Eduardo, FREIRE, João et alii. Revistas, Ideias e Doutrinas - Leituras do Pensamento Contemporâneo. Lisbonne, Livros Horizonte, p. 43-52, 2003.

FREIRE, João. Les anarchistes du Portugal. Paris : Ed. CNT-Région parisienne, 2002.

. Revistas anarquistas portuguesas : entre a política e a cultura. In : REIS, António, LOURENÇO, Eduardo, FREIRE, João et alii. Revistas, Ideias e Doutrinas - Leituras do Pensamento Contemporâneo. Lisbonne, Livros Horizonte, 2003, p. 153-180.

GRAY, John. A Morte da Utopia e o Regresso das Religióes Apocalípticas. Trad. par Freitas e Silva. Lisbonne : Guerra \& Paz « A Ferro \& Fogo », 2008.

JORGE, Ângelo. O amôr sécsual. Amanhã, n² 2, p. 4-6, 15 juin 1909a. . A Fabrica. Amanhã, n 4, p. 13, 15 juil. 1909b.

. A fórmula autoritaria. Amanhã, nº 6, p. 1-5, 15 août 1909c.

LEFEBVRE, Henri. Le Marxisme. 23e éd. Paris : P.U.F. « Que sais-je ? ; n 300 », 2006.

LEITE, Coriolano. Amôr livre. Amanhã, n² 2, p. 10, 15 juin 1909.

MESQUITA, António Pedro. O Pensamento Político Português no Século XIX. Lisbonne : Imprensa Nacional-Casa da Moeda « Temas portugueses », 2006.

PEREIRA, João Carlos Vitorino. Regards lusophones sur l'idéologie et l'utopie dans le discours littéraire. Braga: Vercial, 2011

Irmânia : une utopie libertaire et naturaliste d'Ângelo Jorge. In : PEREIRA, João Carlos Vitorino (dir.). Le déplacement réel ou imaginaire dans le monde lusophone - Migrations, exils et terres d'utopie. Paris : Editions des archives contemporaines, 2013, p. 165-215.

PESSIN, Alain. La rêverie anarchiste (1848-1914). Lyon : Atelier de Création Libertaire, 1999

REIS, José Eduardo. Introdução. In : JORGE, Ângelo. Irmânia. Vila Nova de Famalicão : Quasi Edições « Biblioteca das Utopias », 2004, p. 7-26. 
. Do Espírito da Utopia : Lugares Utópicos e Eutópicos, Tempos Proféticos nas Culturas Literárias Portuguesa e Inglesa. Lisbonne : Fundação Calouste Gulbenkian «Textos Universitários de Ciências Sociais e Humanas »/Fundação para a Ciência e a Tecnologia, 2007.

SOUSA, António Joaquim de. O anarquismo hoje - Problemas e possibilidades de uma prática libertária. Utopia - Revista Anarquista de Cultura e Intervenção, $\mathrm{n}^{\circ} 1$, p. 5-15, avril 1995.

VENTURA, António. Anarquistas, Republicanos e Socialistas em Portugal : As Convergências Possíveis (1892-1910). Lisbonne : Cosmos, 2000.

Submetido em : 11/06/2015

Aceito em: 02/11/2015 